cases(Anon, n.d.) in literature. Affected members in family show variable penetration(Kumar et al., 2009; Herrmann et al., 1975).

Coeliac disease (gluten sensitive enteropathy) is an autoimmune condition that primarily affects the small intestine and occurs in people at any age who become sensitive to a protein called gluten in their diet(Burden et al., 2014, Anon, n.d.).

This is the first reported case of KBG syndrome complicated with recurrent urinary tract infections(UTI), vesicoureteral reflux(VUR), anal stricture, recurrent intussusception and Coeliac disease.

Objectives To show the association of autoimmune disease and intussusception with KBG syndrome.

Methods A 16-year-old girl, diagnosed with KBG syndrome presented with recurrent abdominal pain, loose watery stools and loss of appetite for 1 year.

She presented to the hospital with recurrent UTI due to VUR from the age of 3 weeks up to date and had suffered from difficulties in passing stools from birth. However, an anal stricture was diagnosed and an ileostomy was performed at the age of 5 years. The genetic diagnosis of KBG syndrome was confirmed at the age of 10 years. Percutaneous endoscopic gastrostomy (PEG) was performed to manage feeding difficulties at the age of 12 years and she had a history of several spontaneously resolving intussusception episodes since last year. Intellectual disability, behavioral problems and developmental delay specially speech have been part of this problem(Morel Swols et al., 2017).

Her clinical examination in the recent admission was unremarkable except for pallor and right sided abdominal tenderness. The baseline investigations showed iron deficiency anemia and other causes for failure to thrive were ruled out. In addition to radiological diagnosis(by MRI) of intussusception, the second line investigations for abdominal pain and loose stools showed positive for anti-TTG antibody and which was later confirmed by genetic tests (HLA-DQ2 + HLA-DQ8) for coeliac disease.

Results Following the gluten free diet, her abdominal symptoms has shown improvement.

Conclusions KBG syndrome was diagnosed at a later stage in this patient due to the rareness of the condition. This patient has been suffering from the most of the complications of the disease out of which developmental delay and personality changes, led to delayed diagnosis of the abdominal pathology. The further evaluations with endoscopy procedures has been planned. Associated risk of autoimmune diseases and intussusception with KBG syndrome is yet to be identified.

\section{COMPARISON BETWEEN INFANTS AND OLDER CHILDREN WITH COVID-19 INFECTION}

Kah Wee Teo, Khuen Foong Ng, Srini Bandi. UK

\subsection{6/bmipo-2021-RCPCH.101}

Background Children are less likely to be severely affected or symptomatic from Severe Acute Respiratory Syndrome Coronavirus 2 (SARS-CoV-2) compared to adults. Currently, there is limited evidence on direct comparison of the presentation, clinical features, and managements between infants and older children.
Objectives To understand the difference between the impact of COVID-19 on infants (less than 1 year old) and older children (1 year to 16 years old)

Methods A single-centre retrospective cohort study evaluating children with confirmed COVID-19 who were admitted to University Hospitals of Leicester (UHL), United Kingdom between March to June 2020.

Results Between March to June 2020, a total of 17 children had been hospitalised with subsequent positive SARS-CoV-2 RNA PCR, of which 59\% were infant $(n=10)$. Older children had significantly higher CRP compared to infants. All older children (7/7) had elevated CRP ranging between 8 $\mathrm{mg} / \mathrm{L}$ to $403 \mathrm{mg} / \mathrm{L}$. $71.4 \%$ (5/7) of them had CRP more than $100 \mathrm{mg} / \mathrm{L}$. Among the infants, 50\% (5/10) had normal CRP (CRP $<5 \mathrm{mg} / \mathrm{L}$ ) and 30\% (3/10) had no blood tests taken during admission as they were clinically well. Higher proportion of older children with COVID-19 required admission into paediatric intensive care unit (PICU) or high dependency unit (HDU) and had significantly longer hospital stay compared to infant.

Abstract 187 Table 1 Differences in clinical features, investigation results, management and outcomes between infants and older children with COVID-19.

\begin{tabular}{|c|c|c|c|c|}
\hline & $\begin{array}{l}\text { Infant } \\
\text { (<12 } \\
\text { months) } \\
\mathrm{N}=10\end{array}$ & $\begin{array}{l}\text { Older children } \\
\text { (1-16 years } \\
\text { old) } \\
\mathrm{N}=7\end{array}$ & $p$-value & Statistical test \\
\hline Fever, $\mathbf{n}(\%)$ & $6(67)$ & $3(33)$ & 0.637 & Fisher's Exact \\
\hline $\begin{array}{l}\text { Respiratory symptoms, } \mathrm{n} \\
\text { (\%) }\end{array}$ & $5(63)$ & $3(38)$ & 1 & Fisher's Exact \\
\hline $\begin{array}{l}\text { Gastrointestinal symptoms, } \\
\text { n (\%) }\end{array}$ & $1(25)$ & $3(75)$ & 0.25 & Fisher's Exact \\
\hline Co-morbidities, n (\%) & $1(33)$ & $2(67)$ & 0.537 & Fisher's Exact \\
\hline CRP mg/L, median (range) & $5(5-25)$ & $135(8-403)$ & 0.003 & Mann-Whitney \\
\hline Abnormal CXR, n (\%) & $2(40)$ & $3(60)$ & 1 & Fisher's Exact \\
\hline Antibiotic usage, n (\%) & $5(42)$ & $7(58)$ & 0.044 & Fisher's Exact \\
\hline Oxygen requirement, n (\%) & $1(25)$ & $3(75)$ & 0.25 & Fisher's Exact \\
\hline PICU/HDU admission, n (\%) & $1(20)$ & $4(80)$ & 0.101 & Fisher's Exact \\
\hline $\begin{array}{l}\text { LOS in days, median } \\
\text { (range) }\end{array}$ & $3(1-7)$ & $12(4-22)$ & 0.002 & Mann-Whitney \\
\hline
\end{tabular}

CRP - C-Reactive Protein; CXR - Chest X Ray; HDU - High Dependency Unit; LOS - Length of Stay; PICU - Paediatric Intensive Care Unit

Conclusions Between March and June 2020, hospitalised older children in UHL appeared to be more adversely affected by COVID-19 compared to infants. This may correlate with the emergence of Paediatric Inflammatory Multisystem Syndrome Temporally associated with SARS-CoV-2 (PIMS-TS) and increased incidence of Kawasaki-like disease. Previous findings of infants having more severe disease from COVID-19 by Dong et al might be misleading as it predated the emergence of PIMS-TS and Kawasaki-like disease. The intention of this report is to alert the paediatric community that older children are likely to have a more severe disease when admitted with COVID-19. As this disease is a relatively new entity with evolving clinical picture, clinicians should be open-minded and remain vigilant. 